dose of santonin will immediately reduce the apparently normal performance of the function, together with other consensual phenomena, when they have been dormant for several months, it is entitled to some further distinction in our Pharmacopœia than that of being simply a vermifuge. 1t would be necessary to accept a theory that ovulation could at a certain stage be temporarily suspended, and capable of being immediately accelerated under the influence of certain induced conditions, before we could acknowledge the action of santonin as a true emmenagogue. Manchester.

\section{CASE OF A CRYPTORCHID.}

\section{BY WILLIAM STEER, M.R.C.S.,}

RESIDENT ASSISTAYT MEDICAL OFFICER, ST. OLAVE'S INFIRMARX.

THE connexion between the organs of generation and those of the voice, and the association existent in point of time with their mature development has been always recognised; but how intimate this connexion is, together with the reason why, is not so fully made out. A case therefore where both these sets of organs are rudimentary must contain points of some interest, and a short account of such a one will give matter for reflection (apart from the curiosity that attaches to a condition so rare), and possibly prove of some practical value.

The patient, J. McC- - aged thirty-nine, was admitted into St. Olave's Infirmary in October, 1884, suffering from occasional neuralgic pains in different parts of the thorax, a dry cough, shortness of breath, frequent throbbing at the root of the neck, and pain on swallowing. Aneurism of the arch of the aorta just where it gives off the innominate artery was diagnosed. The man was kept quietly in bed; moderate doses of iodide of potassium were prescribed, and a nonstimulating diet given. The condition of the patient after a couple of months was greatly improved. About this time he complained of pain across his loins, and of having to pass urine as frequently as fifteen or twenty times during the twenty-four hours, hot tea in his case acting as a singularly smart diuretic. Attention being thus drawn to the urinary tract, the following curiously undeveloped condition of those parts was accidentally discovered.

The penis is about an inch in length and a third of an inch in diameter close to the pubes. The prepuce can be easily drawn back, exposing a normally shaped glans. The scrotum resembles in shape and appearance that of an infant, smooth and slightly pink in colour, though differing but little from the rest of the skin, which is singularly white, soft, and clear, there being an entire absence of hair on the trunk and limbs, save a few straggling filaments close around the penis. Passing over the crest of the pubis on either side can be felt a solid cord, about half the thickness of an ordinary cedar pencil, which ends at the upper part of the scrotum in a somewhat flattened fibrous termination, this being the only apology for testes that can be found. Moderate pressure bet ween the finger and thumb gives some pain, but occasions nothing approaching the sensation caused by a like proceeding in a healthy mature testis. With the finger in the rectum no vesiculæ seminales can be felt, nor prostate, neither are any of the ordinary sensations experienced when pressure is made in the region of these organs. The frequency of passing urine points to want of development of the bladder, as the necessity to micturate frequently has existed as long as he can remember, and without other symptoms. On turning towards the larynx and examining through the skin, the trachea is found to be about the average sizeperhaps, if anything, slightly smaller; but the cricoid cartilage is apparently quite natural as to size, prominence, and firmness. The thyroid cartilage, however, presents many marked signs of ill development, it being impossible to distinguish any prominence corresponding to a pomum Adami, nor any notch at the upper border of the cartilage. The larynx appears to be of the proper length and breadth, but gives one the impression of being flattened from before backwards. It is very movable, and the thyroid cartilage is softer than natural. The voice has never broken; it has the ordinary treble quality of a lad's previous to the age of puberty. The mammæ are probably a little larger than usual, with the nipple flat, on a level with the surrounding skin, and of a pinkish colour; as also is the areola. The patient is otherwise well made; is a fairly intelligent man; height 5 ft. 4 in., chest girth 38 in., and exceptionally large around the hips. His hair is weak and thin, and he has commencing cataract in the right eye. He is single, and has apparently never experienced sexual desires.

Rotherhithe, S.E.

\section{A CASE OF DAMAGE TO THE SPINAL ACCESSORY NERVE.}

Bx JOSEPH L. HEWER, F.R.C.S., B.S., M.B.

Mrs. B-, aged sixty-nine, came to me in March, 1885, complaining of a tumour which had been growing out of her back for the last four months. Even beneath her dress a distinct swelling could be seen near the situation of the right scapula, and on uncovering the back it was at once evident that the tumour complained of was the right scapula, which was placed lower than its fellow and stuck out in a most remarkable manner. The right trapezius muscle was extremely atrophied, so that on asking the patient to shrug her shoulders only the left trapezius could be got to act at all, and it appeared that the displacement of the bone was entirely due to the wasting of the muscle on that side. On further examination the right sterno-mastoid was seen to be so wasted that it appeared beneath the skin as a narrow fibrous band, the difference between it and its fellow being most marked. All the other muscles appeared normal. As the patient was muscular, but very thin, she was a good subject for examination. On being questioned, she pointed out a small scar just in front of the right ear at the apex of the anterior triangle, which she said was where a doctor opened an abscess which was deep down in the neck four months ago. Until the formation of this abscess she was quite well, but a few days after it was opened the lump began to form in her back, which gradually got larger; for the last few weeks, however, it had remained stationary; the right arm also got so weak that she could hardly dress herself. I had several opportunities of examining her, but towards the midale of April quite lost sight of her. However, on Aug. 6th, 1885, I again saw her, and was much surprised to find that the right trapezius and sterno-mastoid muscles had apparently recovered their normal bulk, the right scapula being only very slightly more prominent than its fellow and that she was able to use the right arm as well as she ever could.

I think there can be little doubt that the spinal accessory nerve was in some way or other damaged in this patient; it seems almost impossible that it could have been divided in opening the abscess; but it appears more probable that it had become implicated in the products of inflammation, and that thus for a time its function was abolished. What astonished me most was the complete recovery of the patient in so short a time, and that without electricity or treatment of any kind at all. I had told her that she would not get worse, but would certainly get no better, forming this prognosis on the rapid and extreme wasting of the affected muscles and their stationary condition for about a month. The case would appear to me to be a caution against early operation in some cases of supposed division of nerves.

Highbury New Park.

\section{ON A CASE OF NEUROPATHIC PLTCA. BX DINSHA BAMANJI PESTONJI, L.M. \& $\mathrm{S}$. (MASTER.)}

IN October, 1883, a female patient (D. M--), aged about twenty, came to the Dispensary with a peculiar matting of the hair of the head. She said that two days ago, after the cessation of the usual menses, she took a bath and washed her head with soda and warm water. The substance that she applied to the head was supposed to be soda, but she could not say with certainty whether it was soda or anything else. About two hours after the application of the substance to the head, she said that the hair on the back part

1 Read before the Grant College Medical Society of Bombay. 